Recherches en didactique des langues et des cultures

Les cahiers de l'Acedle

\title{
Activité dialogique à l'école : observations sur l'élaboration des significations au sein du dialogue scolaire
}

Catherine Delarue-Breton

\section{OpenEdition}

Édition électronique

URL : http://journals.openedition.org/rdlc/1875

DOI : $10.4000 /$ rdlc. 1875

ISSN : 1958-5772

Éditeur

ACEDLE

\section{Référence électronique}

Catherine Delarue-Breton, « Activité dialogique à l'école : observations sur l'élaboration des significations au sein du dialogue scolaire », Recherches en didactique des langues et des cultures [En ligne], 14-2 | 2017, mis en ligne le 15 juin 2017, consulté le 20 avril 2019. URL : http:// journals.openedition.org/rdlc/1875; DOI : 10.4000/rdlc.1875

Ce document a été généré automatiquement le 20 avril 2019

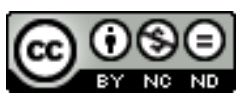

Recherches en didactique des langues et des cultures is licensed under a Creative Commons AttributionNonCommercial-NoDerivatives 4.0 International License 


\title{
Activité dialogique à l'école : observations sur l'élaboration des significations au sein du dialogue scolaire
}

\author{
Catherine Delarue-Breton
}

\section{Introduction}

1 Si la notion de dialogisme, issue notamment des travaux de Bakhtine (1984) et reprise ensuite dans une littérature abondante, fournit un outil d'analyse robuste pour mieux cerner les différents plans du discours, et notamment pour montrer la diversité des situations énonciatives auxquelles est confronté le sujet parlant, elle permet également de contribuer à la définition d'un objet de recherche que nous désignons comme activité dialogique des élèves, et qui participe de la production de significations en classe.

2 Ce sont différents aspects de cette activité dialogique que nous proposons ici de mettre en évidence, et plus particulièrement son caractère hétérogène, puisque les élèves puisent à différentes sources pour élaborer des significations à partir des énoncés qui leur sont soumis.

3 Cette activité silencieuse se manifeste parfois en surface dans le discours en train de se dire, à travers l'irruption de propos incongrus - ou jugés tels par les interlocuteurs - que l'on considère ici, à la suite de Fenoglio (2006), comme évènements d'énonciation.

4 Nous définirons en premier lieu le cadre de l'analyse, en précisant les soubassements théoriques de la recherche et en donnant des indications sur les corpus étudiés; puis nous montrerons, à partir d'extraits de corpus analysés, comment ces évènements d'énonciation permettent de remonter le fil de cette activité dialogique singulière. 


\section{Dialogue, significations, dialogisme : le cadre de l'analyse}

5 L'étude de l'activité dialogique des élèves s'inscrit dans une conception du dialogue au sein duquel des significations, parmi plusieurs possibles, se dessinent (François, 1990); il s'agit donc ici de chercher à mieux comprendre comment le dialogue au sein de la classe, dans sa dimension intersubjective, contribue, pour chacun, à la production de significations intrasubjectives.

\section{Éléments théoriques}

6 Préciser le soubassement théorique de ces analyses invite à revenir rapidement sur le concept de dialogisme, et sur celui d'événement d'énonciation.

\section{Quelques précisions sur l'usage du concept de dialogisme}

7 Le concept de dialogisme permet, depuis Bakhtine, de concevoir le discours comme imprégné des différentes voix qui le traversent. Il contribue de ce point de vue à rompre avec le modèle de l'unité imaginaire du sujet parlant (voir Authier-Revuz, 1984, notamment), mais aussi avec celui de la transparence illusoire du langage; les phénomènes de non coïncidence des mots et des choses, des mots entre eux et du discours à lui-même invitent dès lors à concevoir les ambiguïtés du discours comme des espaces au sein desquels peut s'exercer le jeu des significations. Au delà de ces phénomènes d'inscription de l'altérité dans le discours, qu'ils soient constitutifs du discours, interlocutifs ou interdiscursifs (voir sur ce point notamment Bres \& Nowakowska, 2006), et qui prennent des formes très variées (des voix, des points de vue, des énoncés diffus...), le dialogisme concerne aussi le dialogue entre celui qui parle et sa propre parole; J.Authier-Revuz parle alors d'autodialogisme, J.Bres de dialogisme intralocutif.

Il semble que ce soit à l'intersection de l'interlocutif (dans le dialogue scolaire entre les acteurs), de l'interdiscursif (dans le dialogue entre les discours), et de l'intralocutif (dans le dialogue que le sujet établit avec ses propres conceptions antérieures) que l'on puisse situer l'activité dialogique des élèves en classe. Est donc dialogique aussi la voix qui s'exprime à l'insu du locuteur d'une manière particulière, à son corps défendant (Bres, 2005 : 59). Dans ce cas, l'irruption dans le discours de cette voix refoulée, l'évènement d'énonciation, invite à faire une place à l'Autre en soi-même.

\section{La notion d'évènement d'énonciation}

Pour I. Fenoglio (2006), l'évènement d'énonciation, dont le lapsus est l'exemple le plus pertinent, s'inscrit à la croisée des champs de la subjectivité et de la singularité. La division du subjectif en subjectif et singulier permet à l'auteure de faire apparaître l'opposition entre deux instances du discours, la parole singulière, émanation d'un inconscient non maîtrisable, entrant parfois en conflit avec ce qui est en train d'être dit en discours, qui relève du conscient plus ou moins maîtrisé. La notion d'évènement d'énonciation permet ainsi de saisir, au sein même du discours en train de se dire, quelque chose des cheminements souterrains du sens, différents de ceux qui s'effectuent 
en surface, et qui sont susceptibles de faire obstacle à une production de significations pertinentes pour apprendre à l'école.

Le parti pris au cours de ces analyses (pour plus de détails, voir notamment DelarueBreton, 2012 ; 2014 ; 2016) repose en effet sur le principe que certains propos d'élèves, qui peuvent apparaitre comme incongrus, ne sont pas des propos hors de propos, mais peuvent être interprétés au contraire comme la manifestation d'une production de significations parallèles, et donc révéler quelque chose de la manière dont se nouent les significations dans la psyché. Rapprochée du concept d'évènement d'énonciation tel que défini précédemment, l'incongruité vient ainsi rompre une certaine linéarité discursive, et ce faisant, nous apparaît comme susceptible de traduire l'activité cognitive singulière et intrapsychique des élèves, qui dans le même temps s'inscrit dans un contexte socialement situé (celui de la classe, sous-tendu par la nécessité d'apprendre). Pour autant, bien qu'elle rende manifeste l'activité dialogique singulière d'un élève ici et maintenant (l'auteur de l'incongruité), elle permet aussi la mise au jour de malentendus qui peuvent être aussi le fait d'autres élèves, pourtant demeurés silencieux. Les analyses ici conduites consistent donc à identifier les ruptures dialogales que constituent ces évènements d'énonciation, et à tenter de remonter le fil dialogique qui permet de les interpréter, en rapprochant les propos qui les contiennent d'autres propos, qui se sont tenus précédemment au sein-même de la séance, ou au cours de séances antérieures.

\section{Les corpus étudiés}

11 Les corpus étudiés sont issus de deux séries de séances de classes enregistrées et entièrement transcrites, et constitués l'un par C. Gomila (2007) pour sa thèse de doctorat, l'autre par nous-même.

12 Le premier [désormais corpus CG Lecture] est constitué de quarante séances d'apprentissage de la lecture en classe de $\mathrm{CP}^{1}$ (nous avons exploité une quinzaine d'entre elles), et permet l'étude de la production de significations par les élèves à partir de supports de lecture.

Le second corpus [désormais corpus CDB Dictons] est constitué de vingt-huit séances successives d'interprétation de dictons, également en classe de CP. Ce dispositif original, mis en place dès le début de l'année par l'enseignante, consiste à inaugurer la journée d'école par un rituel. Après quelques propos sur la date et «l'étude de la météo » (nous reprenons les termes employés par la classe), l'échange s'organise plus précisément sur le dicton du jour, fourni par l'enseignante et écrit au tableau. Les élèves sont alors invités, dans un oral collectif semi-dirigé, à en explorer les termes, et à les mettre en relation à la fois entre eux et avec le contexte calendaire, saisonnier ou " météorologique ", et avec les connaissances de chacun ou les connaissances antérieurement acquises par la classe sur le sujet. Les propos des uns et des autres peuvent ainsi faire une très grande diversité d'interprétations de ce qui peut constituer une réponse attendue ou un raisonnement attendu, ce que nous illustrons ci-dessous. 


\section{Trois cas de figure : des significations plus ou moins partagées}

Les trois exemples qui suivent visent à faire valoir différents aspects de l'activité dialogique des élèves en classe, qui donne lieu aussi bien à une production de significations pertinentes que non pertinentes, et qui puise à des sources très diverses. Dans le premier, la production de significations est influencée par les discours qui se sont tenus en amont, lors de la première phase de l'activité (« l'étude de la météo »), qui n'a pourtant pas de rapport direct avec l'activité d'interprétation attendue. Le second, à partir de deux extraits, issus de chacun des deux corpus étudiés, illustre la puissance du discours pédagogique, sans doute souvent sous-estimée, dans le temps des échanges en classe; dans ce cas, la production de signification est influencée par des propos tenus antérieurement - parfois plusieurs jours auparavant - par l'enseignante elle-même, qui n'ont pas non plus de lien direct avec l'activité d'interprétation attendue. Le troisième montre enfin comment l'enseignante parvient à orchestrer le discours de la classe, qui devient dès lors polyphonique : les voix, qu'elles relèvent de l'interaction immédiate ou qu'elles proviennent de moments antérieurs, se répondent davantage.

\section{Le rôle du préconstruit culturel}

Ce premier exemple, issu du second corpus (corpus CDB Dictons), montre, au delà des effets d'échos entre énoncés discontinus, comment un moment récurrent de l'activité peut se constituer en préconstruit culturel (Jodelet, 2006), et dans quelle mesure les significations produites par les individus dans un contexte donné s'inscrivent dans l'expérience antérieurement vécue, entendue comme socialement définie et construite. Chacune de ces séances d'interprétation de dicton ${ }^{2}$ est en effet précédée d'un temps d'observation "météorologique ", nous l'avons dit, qui consiste à observer le ciel et le décrire, à indiquer la température extérieure, et à donner quelques détails sur le temps observé (chute de pluie, neige, vent etc.). Le dicton à étudier ce jour-là est le suivant: Quand arrive la saint Fulbert, dans la campagne tout est vert. Nous citons ici un extrait de la séance, qui comprend les deux temps du dispositif :

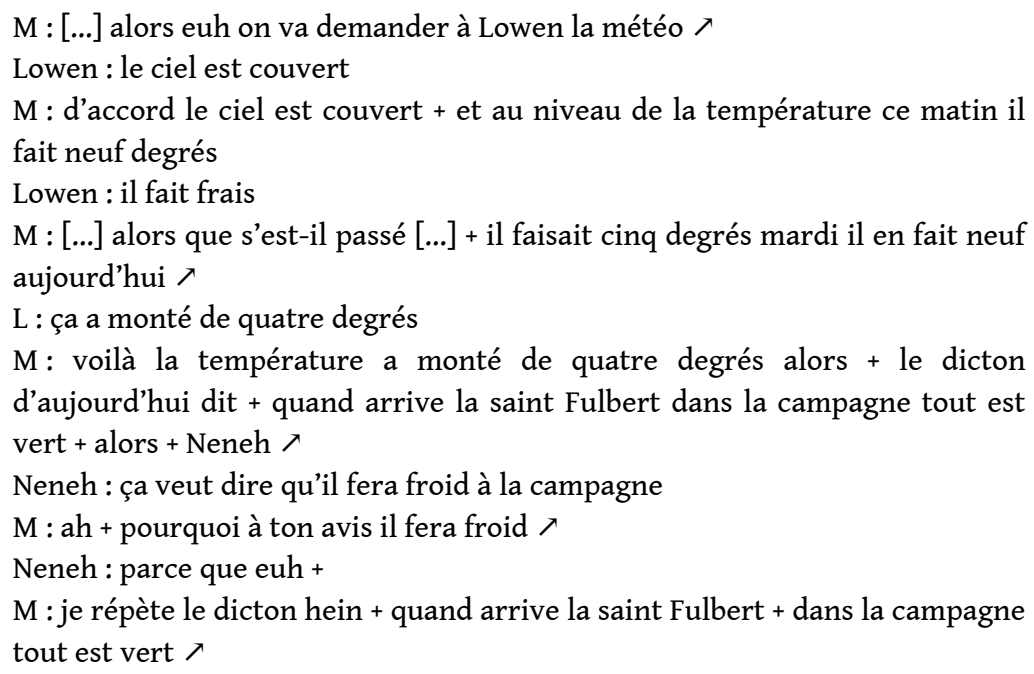


Neneh : à la campagne eh ben il fera beau + parce que euh c'est euh + le printemps

$\mathrm{M}$ : oui mais pourquoi est-ce que tu as pensé qu'il fera froid $\nearrow+$ puisque c'est ce que tu nous as dit en premier $\nearrow$

Neneh : [...] c'est parce que chez nous il fait froid

$\mathrm{M}$ : ça veut dire quoi chez nous chez qui $\nearrow$

Neneh : à Paris

$\mathrm{M}:$ ah oui mais + là est-ce que Paris est à la campagne $>$

LL : non

$\mathrm{M}$ : non alors on vous dit + dans la campagne tout est vert Morgane $\nearrow[. .$.

Morgane : ça veut dire que + il fera froid

$\mathrm{M}$ : ben on vient de faire cette réponse avec Neneh + tu n'as pas vraiment

écouté il me semble + Nour $\nearrow$

Nour : ça veut dire que c'est le printemps

$\mathrm{M}:$ ah oui et pourquoi $\nearrow$

Nour : parce que tout est vert [...] [Corpus CDB Dictons]

Cet extrait, emblématique du déroulement des vingt-huit séances du second corpus, donne à voir d'emblée dans quelle mesure la production de significations est influencée simultanément par le discours environnant, mais aussi par le dispositif lui-même, dont certaines phases peuvent en effet tenir lieu de préconstruit culturel : Neneh suggère ainsi dans un premier temps, dans une forme de dialogisme que l'on peut analyser comme interdiscursive, que le dicton évoque la froidure à venir, non parce que le sens des termes du dicton l'y invite, mais parce que l'observation météorologique l'y invite. La classe a ainsi pu constater que malgré l'augmentation de quatre degrés de la température, il faisait frais (formule attestée dans le discours de l'enseignante), ce qui la conduit à suggérer que le dicton parle peut-être du froid, propos qui sera ensuite repris par Morgane. Au niveau interlocutif, l'intervention de l'enseignante concernant l'opposition campagne/Paris ne permet pas de mettre en évidence les effets d'écho entre ces différents plans du discours, à l'origine de ces significations non pertinentes dans le contexte, et donc ne contribue pas au dialogue intralocutif qui permettrait aux élèves de se déplacer, et de produire les significations attendues.

Si ce corpus original est sans doute assez peu représentatif des séances ordinaires de la classe de $\mathrm{CP}$, il permet cependant de saisir quelque chose du fonctionnement psychique d'élèves confrontés à l'aporie, cas de figure très fréquent dans ce corpus particulier, mais récurrent aussi dans d'autres types d'activités; nous entendons par aporie une situation au cours de laquelle les élèves, complètement bloqués dans la production de signification, après plusieurs tentatives infructueuses pour élaborer le sens du propos, en viennent à tenter une réponse fermement validée dans un autre contexte, au risque de produire un énoncé insensé dans le contexte présent.

17 Au-delà du préconstruit culturel toutefois, on observe qu'une autre dimension influence fortement la production de signification par les élèves, c'est celle de la parole enseignante.

\section{Le fil rouge de la pertinence : puissance de la parole enseignante}

Les deux exemples ci-dessous visent ainsi à montrer quelque chose des effets intrasubjectifs de la parole de l'enseignant, en lien avec sa position de surénonciateur (Rabatel, 2005), et indépendamment de la variété des contextes didactiques dans lesquels se déploie ce dialogue scolaire. 
Le dicton à interpréter ce jour-là est le suivant : À la saint Pierre Damien, l'hiver reprend ou s'éteint. Les élèves peinent à comprendre, compte tenu de la difficulté considérable du dicton pour des élèves de cet âge-là (voir Delarue-Breton, 2016), que si l'hiver reprend, il fera à nouveau froid, tandis que s'il s'éteint, il fera plus chaud. Voici un extrait des échanges :

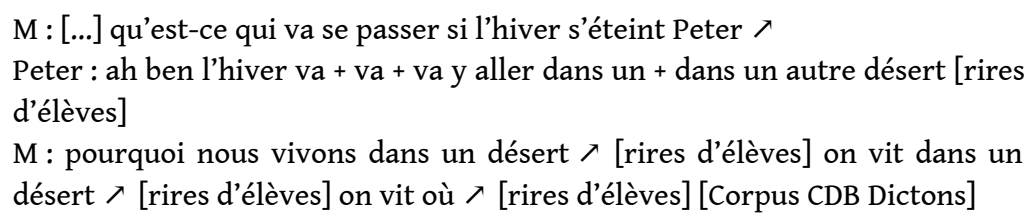

Ici, Peter provoque l'hilarité de ses camarades (ce qui atteste formellement du caractère incongru du propos tel qu'il est perçu par la classe) en évoquant ce que ces derniers et l'enseignante ont entendu comme le désert. L'étude attentive du passage et de l'ensemble de la séance en amont du propos de Peter, afin de tenter de saisir le fil dialogique de son cheminement, nous a permis d'identifier un énoncé produit par l'enseignante au cours de la même séance, rappelant pourquoi, selon elle, les desserts de l'hiver (et non les déserts) sont ronds et jaunes, énoncé susceptible à nos yeux de faire écho avec le propos de Peter. Le passage se situe juste après l'examen météorologique qui inaugure chacune des journées de classe, et qui a permis d'affirmer que le ciel était couvert:

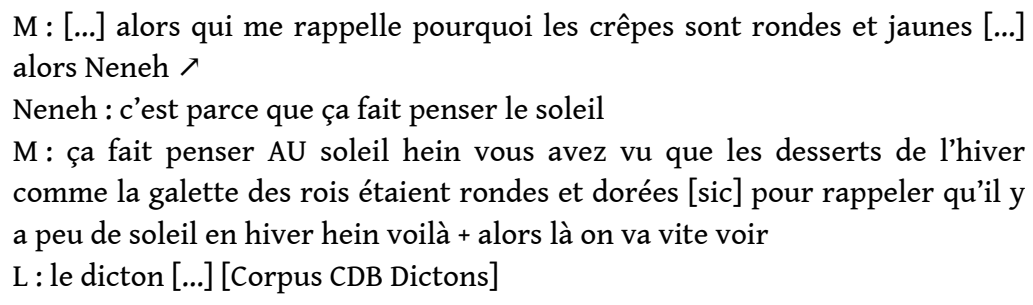

21 À ce moment, l'hiver est très clairement associé dans les propos de l'enseignante à l'absence de soleil ; lorsque la classe en vient un peu plus tard à expliciter le sens de la proposition l'hiver s'éteint, ce qui suppose dans le contexte que le soleil revienne, on peut considérer que Peter, dans un cheminement dialogique enraciné dans les propos du surénonciateur que représente ici l'enseignante, a privilégié un mode de dire en partie métaphorique, que l'on peut traduire ainsi :

Peter : ah ben l'hiver va + va + va y aller dans un + dans un autre désert $=*$ si l'hiver s'en va, on va aller vers un autre dessert [Corpus CDB Dictons]

Si l'on suit cette analyse, loin d'être un propos insensé, le propos de Peter atteste en surface d'une production de significations qui chemine silencieusement, du moins jusqu'à cette manifestation singulière. On peut sans doute considérer que ce type d'évènement d'énonciation est plus fréquent dans le second corpus, parce que les objets d'étude que sont les dictons sont très opaques pour ces jeunes élèves, et que l'aporie, qui favorise selon nous ce type de cheminement, y est d'autant plus fréquente, elle aussi. On le rencontre cependant aussi dans le premier corpus, constitué de séances bien plus représentatives de ce qui se fait en classe de $\mathrm{CP}$. En voici un exemple; il s'agit ici de rappeler les constituants typographiques d'une liste :

M tiens je vais redemander euh Dylan dis-moi pourquoi ce texte est une liste $\nearrow$ pourquoi $\nearrow$ 
Dylan parce qu'il y a beaucoup de dessins [...]

M non pas du tout tu n'as pas écouté ce qu'on vient de dire alors tu vas bien ouvrir tes oreilles et on va demander à un autre copain Tristan pourquoi estce que ce texte c'est une liste $\nearrow$

Tristan parce qu'il y a des tirets

$\mathrm{M}$ parce qu'il y a des petits tirets en début de ligne très bien qu'est-ce qu'il y

a d'autre $\nearrow$ levez le doigt ceux qui veulent dire oui

Caroline il y a un titre

$\mathrm{M}$ il y a un titre très bien dernière chose qu'est-ce que c'est ça $\nearrow$ il y a des $\nearrow$

Caroline colonnes

$\mathrm{M}$ voilà il y a une colonne de mots ici [Corpus CG Lecture]

lan semble n'avoir pas écouté, et l'enseignante le lui reproche ; pourtant, l'étude du début de la séance laisse apparaître des énoncés qui font effectivement référence à des dessins (nous soulignons les passages susceptibles d'avoir influencé la production de signification de Dylan) :

M bien alors vous avez vu le texte au tableau alors je vous rappelle que le petit texte que vous avez sur votre bureau je l'ai agrandi ici au tableau [...] comme ça vous le voyez bien ce texte est-ce que vous êtes d'accord que ce que j'ai écrit là et là ça correspond à votre texte $\nearrow$

LL oui

$M$ oui très bien qu'est-ce qu'il manque simplement $\lambda$ ici en bas qu'est-ce

qu'il manque $\nearrow$

Caroline les dessins

$M$ les petits dessins très bien alors est-ce que quelqu'un se souvient comment s'appelle ce texte $\nearrow$ on lève le doigt si on veut le dire Caroline $\nearrow$ Caroline une liste

$M$ c'est une liste très bien Caroline [Corpus CG Lecture] d'énonciation : proximité des termes, mais souvent aussi reprise partielle d'une tournure 
syntaxique ou d'une locution. Toutefois, ce qui est plus remarquable, c'est la présence généralement associée au propos source de ce que nous appelons des propos de ponctuation , qui valident la proposition de l'élève en ayant à son égard des propos élogieux (très bien), ou viennent en marquer fermement la pertinence (voilà). Dans l'exemple précédemment cité, Dylan a pu associer la liste et les dessins parce que ce rapprochement dans un autre contexte a permis à Caroline de bénéficier à deux reprises des éloges de l'enseignante (très bien [...] très bien Caroline). On peut donc sans doute affirmer ici que le fil rouge de la pertinence, quand les élèves sont confrontés à l'aporie, réside davantage dans la parole validée, dès lors susceptible de valoir en soi, voire dans l'absolu, et non en contexte, que dans la parole qui fait sens.

Si l'étude des évènements d'énonciation qui font irruption dans le dialogue scolaire permet d'apercevoir des cheminements singuliers, celui-ci est cependant susceptible de contribuer également à une production de significations partagées.

\section{Des significations partagées}

Bautier et Rochex (2004) ont mis en évidence que le caractère conjoint des activités en classe, impliquant l'ensemble des élèves, était loin d'entraîner une production de significations partagées ; pour autant, ces significations sont partagées dans bien des cas, et il nous semble nécessaire de comprendre également en quoi le dialogue scolaire contribue à leur élaboration. Cette question des significations partagées nous apparaît ainsi indissociable de la question du discours collectif, susceptible d'impliquer l'ensemble des élèves dans les temps d'approximation successive aussi bien que dans les temps de stabilisation du savoir.

L'étude de différentes séances de classe, issues du premier corpus, dont fait partie l'extrait présenté ci-dessous, permet de constater que les échanges dialogaux, constituant un discours réellement collectif, contribuent à une lecture pertinente du texte proposé, en convoquant tantôt des objets du monde (Edwige, par exemple, qui s'appuie sur l'illustration du texte pour comprendre que le mot moustache désigne la moustache de la souris), tantôt des objets de savoirs (Carmen, par exemple, qui s'appuie sur la segmentation en syllabes, et reconnait le son [mu] à partir du graphème "mou»). Ce discours collectif est par ailleurs fermement arbitré par l'enseignante, qui invite les élèves d'une part à s'écouter et prendre en compte les apports d'autrui, et d'autre part à adopter une posture de distanciation de l'ordre du métacognitif - médiate donc - vis-à-vis de ces propos, en sollicitant les élèves pour qu'ils expliquent comment ils ont pu reconnaitre tel ou tel mot. L'analyse du discours pédagogique permet donc de mieux comprendre le rôle majeur de l'enseignante - y compris dans ses dimensions énonciatives fines - dans l'aide apportée aux élèves. Le passage lu est le suivant : J'ai besoin des poils de ta moustache pour ma prochaine potion magique. Cédric commence à lire :

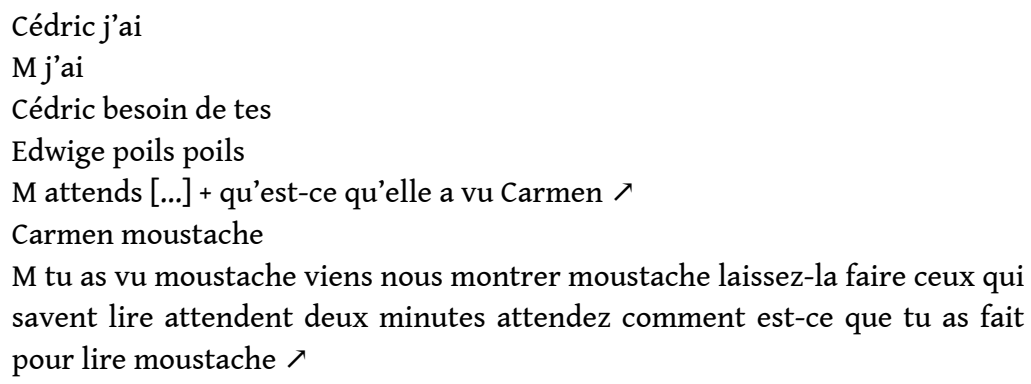


Cédric j'ai besoin de tes moustaches

$M$ pourquoi est-ce que tu penses + c'était

Carmen parce qu'on connait [mu]

M on connaît [mu] bon d'accord ça on le connaît

Michaël je sais on connait encore tous les morceaux de mots

M c'est comme ça que tu as su lire moustache $\nearrow$ est-ce que ça peut être

moustache $\nearrow$ est-ce qu'il y a de fortes chances qu'on trouve le mot moustache $\nearrow$

LL oui

M pourquoi $\nearrow$

Edwige parce que c'est les moustaches qu'on lui a coupées

$M$ et qu'est-ce qui vous fait dire ça $\nearrow$ parce que $\nearrow$

Edwige parce que dans l'image on voit qu'elle arrache les moustaches

M dans l'image on voit alors moi je vais écrire ce que vous m'avez dit

[l'enseignante écrit le mot au tableau] c'est ça $\nearrow$

Morgan oui [...]

Aurore j'ai besoin des poils de ta moustache pour ma prochaine potion magique

$\mathrm{M}$ oh là là + là il y a plein de mots peut-être un petit peu difficiles alors j'aimerais quand même qu'Aurore nous dise + comment est-ce que tu as fait pour lire besoin $>[. .$.

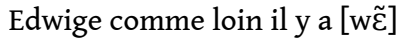

$\mathrm{M}$ alors loin j'écris loin

Cyril il y a $[b ø]$ de octobre tu enlèves le $[\varepsilon r]$ ça fait [be]

Edwige lion

$\mathrm{M}$ ah attends elle m'a dit lion alors j'écris lion [...]

L c'est l'inverse

$\mathrm{M}$ c'est l'inverse attention là besoin loin là c'est lion [...]

Michaël dans besoin on connaît [bø]

$\mathrm{M}$ on connait $[\mathrm{b} ø]$ où on connait $[\mathrm{b} ø] /$ arrête-toi Edwige

Michaël comme dans le classement 13

Edwige beaucoup beaucoup ouais

M euh potion comment est ce qu'ils ont fait pour lire potion $\nearrow$

Laurent on connait [po]

M on connait [po] d'accord

Laurent et il y a + il y a [jõ]

Edwige comme lion

$\mathrm{M}$ ah voilà alors là maintenant d'accord potion je vais le mettre là mais cependant regardez

Laurent maîtresse qu'est ce que tu as mis ça se lit [sjõ] et tu as dit que là c'est lion

M là ça se lit [sjõ] et comment c'est écrit $\nearrow$

L comme poisson

$\mathrm{L}$ avec un [te]

$\mathrm{M}$ avec un [te] [Corpus CG Lecture]

Dans cet extrait, on peut observer l'apport personnel de certains élèves, qui convoquent spontanément (bien que cette spontanéité relève probablement davantage d'une habitude de travail incorporée que d'une spontanéité à proprement parler, on pourrait donc dire de leur propre mouvement) des éléments issus d'ailleurs, comme la référence au classement 13 de Michaël, ou l'exemple du mot lion proposé par Edwige, qui a d'ailleurs précédemment reconnu le mot joli en se référant à un autre texte lu antérieurement dans lequel il figurait également.

Mais l'élaboration du sens du texte repose aussi en grande partie, et c'est là ce qui nous intéresse particulièrement, sur l'apport d'un nombre d'élèves moins diserts qui se lancent 
plus timidement dans l'aventure, ou sont sollicités par l'enseignante (qui orchestre donc ici la pluralité des voix) et contribuent de ce fait collectivement aux approximations successives qui permettent d'avancer. Le jeu de pronoms employés par l'enseignante notamment, impliquant l'ensemble du groupe est révélateur à cet égard: loin d'engager un échange duel avec l'interlocuteur sollicité (ou qui a pris la parole de son propre mouvement), ce que nous avons pourtant observé dans bien des séances, celle-ci intègre la part duelle du dialogue (je, tu) dans un dialogue collectif (on/nous, vous), dont chacun est partie prenante.

31 Ainsi, l'échange avec Carmen est emblématique : " $\boldsymbol{t u}$ as vu moustache viens nous montrer moustache", puis "c'est comme ça que tu as su lire moustache [...] est-ce qu'il y a de fortes chances qu'on trouve le mot moustache", et enfin je vais écrire ce que vous m'avez dit". L'usage du tableau, de même que les affichages (classement 13), participe de l'élaboration de ce discours collectif, qui est donc un discours plurisémiotisé. Enfin, elle invite les élèves plus avancés à différer leur prise de parole, tout en acceptant dans le même temps leur contribution.

L'extrait est ponctué de propos intégrant la parole de l'un dans le dialogue du groupe: viens nous montrer, ça on le connait, qu'est-ce qui vous fait dire ça,j'aimerais qu'Aurore nous dise, regardez, etc. On note que huit élèves au moins (d'autres ne sont pas identifiés) interviennent dans l'échange, qui ne dure pourtant que quelques minutes. Il ne s'agit pas ici de dire que la participation de tous est nécessaire en soi (celle-ci, souvent illusoire, étant loin d'être suffisante pour garantir un quelconque apprentissage), mais on observe que ces huit élèves contribuent effectivement à l'élaboration du sens du passage, et à ce titre, on peut considérer que leurs interventions participent aussi de l'élaboration du discours collectif, qui fait cruellement défaut dans certaines classes, quand bien même la plupart des élèves parle.

\section{Conclusion}

L'étude de ces cheminements dialogiques invite tout d'abord à mettre en avant que les sources auxquelles puisent les élèves pour produire des significations sont à considérer bien au delà du contexte ou cotexte immédiat dans lequel le propos s'inscrit (l'alternance des tours de parole). Ce constat rejoint ce que Salazar-Orvig et Grossen (2008) évoquent du dialogisme dans le dialogal et propose une nouvelle illustration de la manière dont se constitue le dialogisme interne dans la dynamique même de l'échange, ce qui vient confirmer que l'étude du singulier peut, à certaines conditions, dire quelque chose de phénomènes récurrents. On peut ainsi parler de dialogisme interne pour évoquer ces effets d'échos entre les énoncés de Dylan et de Caroline, qui sont discontinus, mais s'inscrivent dans la séance même.

Ces cheminements dialogiques sont par ailleurs fortement influencés par la manière dont les propos des uns et des autres sont validés par le surénonciateur que représente l'enseignant, indépendamment du contexte dans lequel ils s'inscrivent: un propos fermement validé, qui fait l'objet d'éloge dans un contexte donné, peut devenir un propos pertinent en soi ; ainsi, pour Dylan par exemple, il y a lieu de tenter la réponse (pourtant incongrue) dessins pour désigner les constituants typographiques d'une liste, parce que dessin a été fermement validé antérieurement (en l'occurrence, au sein de la même séance, mais il aurait pu en être autrement). 
Ces descriptions de l'activité dialogique des élèves permettent ainsi de déduire certaines nécessités du discours enseignant, et notamment celle de l'orchestration d'un discours réellement collectif (c'est-à-dire qui ne se réduise pas à une pluralité d'échanges duels), impliquant l'ensemble des élèves, et visant l'élaboration d'un texte de savoir. On observe en effet que lorsque l'enseignante se saisit, au niveau interlocutif, de phénomènes dialogiques interdiscursifs individuels, cela contribue à favoriser une production de signification collective pertinente.

Si l'on considère à la suite de Bres (2005) que le dialogisme renvoie à une pluralité de voix non maitrisées, et la polyphonie à une pluralité de voix maitrisées il y a donc lieu de considérer que la visée de l'activité discursive de l'enseignant en classe est notamment de transformer les phénomènes dialogiques (où les effets d'échos entre énoncés sont aléatoires et inexploités), ou du moins certains d'entre eux, en phénomènes polyphoniques (où les effets d'échos entre énoncés peuvent être ressaisis et partagés). La ressaisie des phénomènes d'écho entre les énoncés de Caroline et de Dylan, par exemple, pourrait permettre à Dylan, mais aussi sans doute à d'autres élèves demeurés silencieux, de ressaisir les différents objets évoqués (dessins, tirets, colonnes etc.) dans une orchestration du propos qui pourrait contribuer, selon nous, à l'unification de l'expérience.

\section{BIBLIOGRAPHIE}

Authier-Revuz, J. (1984). « Hétérogénéité(s) énonciative(s) ». Langages, n73. pp. 98-111.

Bakhtine, M. (1984). Esthétique de la création verbale. Traduction française par A. Aucouturier. Paris : Gallimard.

Bautier, É. \& Rochex, J.-Y. (2004). « Activité conjointe ne signifie pas significations partagées ». In Moro, C. \& Rickenmann, R. [éds.], Situations éducatives et significations. Bruxelles : De Boeck. pp. 199-220.

Bres, J. (2005). « Savoir de quoi on parle : dialogue, dialogal, dialogique; dialogisme, polyphonie... ». In Bres, J., Haillet, P.-P., Mellet, S., Nolke, H., Rosier, L. (dir.). Actes du colloque de Cerisy. Dialogisme et polyphonie. Approches linguistiques. Bruxelles : De Boeck-Duculot. pp. 47-61.

Bres, J. \& Nowakowska, A. (2006). « Dialogisme : du principe à la matérialité discursive ». Recherches linguistiques, $\mathrm{n}^{\circ} 28 . p p$. 21-48.

Delarue-Breton, C. (2012). Discours scolaire et paradoxe. Louvain : Academia-L'Harmattan.

Delarue-Breton, C. (2014). « Dialogue scolaire, dialogisme et évènements d'énonciation : malentendus et in-entendus au sein de la classe ». Études de Linguistique Appliquée, n 173. pp. 87-97.

Delarue-Breton, C. (2016). « Le dialogal et le dialogique : quelques remarques sur les heurs et malheurs de l'interaction verbale en classe de français ». In Neveu, F., Bergounioux, G., Côté, M.H., Fournier, J.-M., Hriba, L. \& Prévost, S. (éds.). Actes du Vème Congrès Mondial de Linguistique Française, vol. n²7. http://dx.doi.org/10.1051/shsconf/20162707007. 
Fenoglio, I. (2006). «L'intime étrangeté de la langue », Langage et inconscient, vol. 2. pp. 41-66. François, F. (1990). « Dialogues, jeux de langage et espace discursif chez l'enfant jeune et moins jeune ». In François, F. (dir.). La communication inégale. Heurs et malheurs de l'interaction verbale. Neuchâtel et Paris : Delachaux \& Niestlé. pp. 33-111.

Gomila, C. (2007). Le discours métalinguistique de la classe de lecture : comment les enseignants de cours préparatoires et leurs élèves qui apprennent à lire parlent du langage. Thèse pour le doctorat en sciences du langage, sous la direction de S. Branca-Rosoff, Université Paris 3.

Jodelet, D. (2006). « Place de l'expérience vécue dans le processus de formation des représentations sociales ». In Hass, V. (dir.). Les savoirs du quotidien. Transmissions, appropriations, représentations. Rennes : Les Presses Universitaires de Rennes. pp. 235-255.

Rabatel, A. (2005). « Les postures énonciatives dans la coconstruction dialogique des points de vues : coénonciation, surénonciation, sousénonciation ». In Bres, J., Haillet, P.-P., Mellet, S., Nolke, H. \& Rosier, L. (dir.). Actes du colloque de Cerisy : Dialogisme et polyphonie. Approches linguistiques. Bruxelles : De Boeck-Duculot. pp. 95-110.

Salazar-Orvig, A. \& Grossen M. (2008). « Le dialogisme dans l'entretien clinique ». Langage et société, $\mathrm{n}^{\circ} 123$. pp. 37-52.

\section{NOTES}

1. $C P$ : Cours Préparatoire, première classe de l'école élémentaire française, accueillant des élèves de 6-7 ans.

2. Le code de transcription utilisé est inspiré du Groupe d'Aixois de Recherche en Syntaxe (GARS). $M$ désigne l'enseignante, $L L$ désigne plusieurs élèves, $L$ désigne un élève non identifié, l'emploi d'un prénom renvoie à un élève identifié ; les pauses brèves sont marquées par le signe +; les mots entre crochets droit sont ajoutés par le transcripteur; le signe $\triangle$ indique l'expression d'une attente, à l'endroit des élèves, de la part de l'enseignante ; les mots en caractères droits indiquent une reprise autonymique des termes du dicton étudié. Les syllabes en majuscules indiquent un appui intonatif sur la syllabe; l'alphabet phonétique international (API) a été utilisé pour transcrire les phonèmes épelés ; enfin, [...] indique une coupure effectuée dans le texte transcrit.

\section{RÉSUMÉS}

L'activité dialogique des élèves en classe, entendue comme activité psychique et intellectuelle visant la mise en relation d'objets divers (d'objets de savoir notamment), est difficilement observable, a fortiori difficilement saisissable dans sa linéarité ou son homogénéité. L'étude de cette activité intrasubjective, à partir de l'irruption de certains évènements d'énonciation à la surface du discours de la classe, permet de mieux comprendre certains cheminements en apparence incohérents, qui font pourtant sens quand on parvient à établir des liens entre les différents contextes qui constituent le quotidien des élèves. 
The dialogical activity of the pupils in class, understood as the psychic and intellectual activity aiming at the getting in touch of diverse objects (of objects of knowledge in particular), is hard to observe, especially as linear or homogeneous activity. The study of this intrasubjective activity, on the basis of the appearance, to the surface of the speech, of events of statement, allows to understood some progressions apparently inconsistent. These progressions are however meaningfull when we succeed in establishing links between the ordinary contexts of the pupils.

\section{INDEX}

Mots-clés : activité dialogique, évènements d'énonciation, dialogue, significations

Keywords : dialogical activity, event of statement, dialogue, meanings

\section{AUTEUR}

\section{CATHERINE DELARUE-BRETON}

Université Paris Est Créteil - Laboratoire CIRCEFT-EA4384

Catherine Delarue-Breton est maitre de conférences HDR au laboratoire CIRCEFT-Escol (Université Paris 8 et Paris Est Créteil), et en poste à l'Université de Paris Est Créteil, ÉSPÉ de l'académie de Créteil. Ses travaux visent notamment la description formelle du dialogue scolaire, et une meilleure compréhension des significations produites par les élèves du primaire en classe. catherine.delarue-breton [at] u-pec.fr

http://www.circeft.org 